\title{
Development of an Industrial Internet of Things Suite for Smart Factory towards Re-industrialization in Hong Kong
}

\author{
C.K.M. Lee*, S.Z. Zhang \\ Department of Industrial and Systems Engineering \\ The Hong Kong Polytechnic University \\ Hong Kong, China \\ ckm.lee@ polyu.edu.hk \\ shuzhu.zhang@connect.polyu.hk
}

\begin{abstract}
Re-industrialization, which supports industrial upgrading and transformation, promotes smart production and high value-added manufacturing processes, and helps to create new momentum for the economic growth of Hong Kong. Under the current situation, industrialists encounter several challenges to achieve re-industrialization. First, the cost and technical thresholds for industrialists to leverage emerging technologies are high. Second, there are huge quantities and numerous types of Internet of Things (IoT) devices in smart factories, warehouses and offices. The enormous extents of data exchange and communication, management, monitoring and control of IoT devices as well as the establishment and maintenance of a reliable cloud platform hinder industrialists to implement an integrated smart production management. Therefore, to achieve reindustrialization, upgrade and optimize advanced manufacturing processes in Hong Kong, an Industrial Internet of Things (IIoT) Suite consisting of a Micro-services-based IIoT Cloud Platform and IIoT-based Smart Hub is proposed, which helps to materialize re-industrialization in Hong Kong and to conduct industrial upgrading and transformation to achieve smart production and high value-added manufacturing processes.
\end{abstract}

Keywords-Re-industrialization; Internet of Things; Industrial Internet of Things; Cloud Platform; Smart Hub

\section{INTRODUCTION}

According to the 2016 Policy Address, Hong Kong needs to discover momentums for economic growth [1]. Reindustrialization is one of the potential areas to achieve this goal. To grasp the chances brought by re-industrialization, the Innovation and Technology Bureau (ITB) is constructed and re-industrialization is identified as one of the nine directions and work priorities [2, 3]. ITB attempts to investigate the Internet of Things (IoT) to support industrial upgrading and transformation, as well as promotes smart production and high value-added manufacturing processes. In addition to the government policy, Hong Kong industrialists have been starting to move production from mainland China back to Hong Kong in considering of the appreciating Chinese currency, the rising labor wage rates and the decreasing supply of labor. The unstable supply of labor drives industrialists to increase automation in companies so as to reduce reliance on labor and to retain customers by offering innovative solutions or value-added services [4]. However, the major challenge of finding the proper ways to achieve industrial upgrading and transformation as well as to move towards high-value added production is not yet addressed.

Industrial Internet of Things (IIoT) has been introduced recently as the adoption of IoT in manufacturing or industrial domains. Because of the advanced development of IIoT, the industrial sector has undertaken a new paradigm shift from traditional manufacturing to contemporary industry 4.0 manufacturing with the support of Cyber-Physical Systems (CPS). However, industrialists encounter several challenges to achieve re-industrialization. First, the cost and technical threshold for industrialists to leverage emerging technologies are high. In the current situation, most of the manufacturing processes in Hong Kong and Pearl River Delta Region are semi-automated processes. Second, to facilitate the development of IIoT, various IoT devices are acquired and installed gradually to upgrade and transform existing processes. Third, as there are huge quantities and numerous types of IoT devices in the smart factories, warehouses and offices, the voluminous data exchange and communication, management, monitoring and control of IoT devices become the challenges to industrialists due to inadequate technical knowledge and high start-up and maintenance costs. In such situation, it is vital to provide a simple and convenient way to connect and communicate with existing IoT devices. Apart from managing, monitoring and controlling the IoT devices, a sustainable approach to maintenance and advancement is also important to industrialists.

Moreover, using IoT devices, each with its individual management platform without interconnection makes it difficult for industrialists to manage, monitor and control various IoT devices. A single and reliable cloud platform is preferred by industrialists. Beyond optimizing platform usage as well as conducting data analysis and reporting, the cloud platform is sustainable for future expansion and upgrades. Industrialists can also transfer the useful statistical data or information from the platform to other Information Technology (IT) and/or Operation Technology (OT) systems for analyses to inform risk management and process improvement so as to implement smart production and high-value added productions. 
In this research, in order to address the industrial challenges, achieve re-industrialization and future innovation and technology development in Hong Kong particularly in manufacturing and business processes, an IIoT Suite consisting of a Micro-services-based IIoT Cloud Platform and an IIoTbased Smart Hub is proposed. The IIoT Suite provides an information and operational infrastructure for industrialists to materialize re-industrialization in Hong Kong and to conduct industrial upgrading and transformation to achieve smart production and high value-added manufacturing processes.

\section{INDUSTRY 4.0 AND CPS}

\section{A. Industry 4.0}

Industry 4.0 has attracted numerous attentions from academic researchers and industrial practitioners ever since the German federal government announced industry 4.0 as one of the key initiatives of its high-tech strategy in 2011 [5]. Smart factory in the context of industry 4.0 is becoming a new manufacturing pattern with latest technologies. Table 1 illustrates a comparison of today's factory and industry 4.0 factory.

TABLE I.

Comparison of today's factory and industry 4.0 factory [6]

\begin{tabular}{|c|c|c|c|}
\hline & & Attributes & Technologies \\
\hline \multirow[b]{2}{*}{$\begin{array}{l}\text { Component } \\
\text { Sensor }\end{array}$} & $\begin{array}{l}\text { Today's } \\
\text { factory }\end{array}$ & Precision & $\begin{array}{l}\text { Smart sensors } \\
\text { Fault detection }\end{array}$ \\
\hline & $\begin{array}{l}\text { Industry } \\
4.0 \\
\text { factory }\end{array}$ & $\begin{array}{l}\text { Self-aware } \\
\text { Self-predict }\end{array}$ & $\begin{array}{l}\text { Degradation monitoring } \\
\text { Remaining useful life } \\
\text { prediction }\end{array}$ \\
\hline \multirow{2}{*}{$\begin{array}{l}\text { Machine } \\
\text { Controller }\end{array}$} & $\begin{array}{l}\text { Today's } \\
\text { factory }\end{array}$ & $\begin{array}{l}\text { Production } \\
\text { rate } \\
\text { Performance } \\
\end{array}$ & $\begin{array}{l}\text { Condition-based } \\
\text { monitoring \& diagnostics }\end{array}$ \\
\hline & $\begin{array}{l}\text { Industry } \\
4.0 \\
\text { factory }\end{array}$ & $\begin{array}{l}\text { Self-aware } \\
\text { Self-predict } \\
\text { Self-compare } \\
\end{array}$ & $\begin{array}{l}\text { Up time with predictive } \\
\text { health monitoring }\end{array}$ \\
\hline \multirow{2}{*}{$\begin{array}{l}\text { Production } \\
\text { system } \\
\text { Network } \\
\text { system }\end{array}$} & $\begin{array}{l}\text { Today's } \\
\text { factory }\end{array}$ & $\begin{array}{l}\text { Productivity } \\
\text { OEE }\end{array}$ & $\begin{array}{l}\text { Lean operations: work } \\
\text { and waste reduction }\end{array}$ \\
\hline & $\begin{array}{l}\text { Industry } \\
4.0 \\
\text { factory }\end{array}$ & $\begin{array}{l}\text { Self-configure } \\
\text { Self-maintain } \\
\text { Self-organize } \\
\end{array}$ & Worry-free productivity \\
\hline
\end{tabular}

\section{B. $C P S$}

CPS relies on the newest and foreseeable further developments of Computer Science (CS), Information and Communication technologies (ICT) and Manufacturing Science and Technology (MST). There is increasing enthusiasm in exploiting the latest technologies for the CPS in order to enhance productivity and product quality, among which, IoT is the most essential and fundamental technology that enable the CPS.

The application of CPS in manufacturing has been studies by numerous researchers. For example, Mikusz [7] regarded CPS as an industrial software-product-service system in view of the perspectives from solution, value chain and software part and proposed a structure of CPS as illustrated in Fig. 1. Monostori [8] introduced a Cyber-Physical Production Systems (CPPSs) considering the interplay between computer science, information and communication technology and manufacturing automation. Lee, Bagheri [6] introduced a 5C CPS structure to guide the development and deployment of CPS in manufacturing domain, which comprises smart connection, data-to-information conversion, cyber, cognition and configuration. And in the cyber level, they introduced a concept of time machine to conduct the Prognostic and Health Management (PHM), which consists of three steps, i.e., snapshot collection, similarity identification and synthesis optimized future operation.

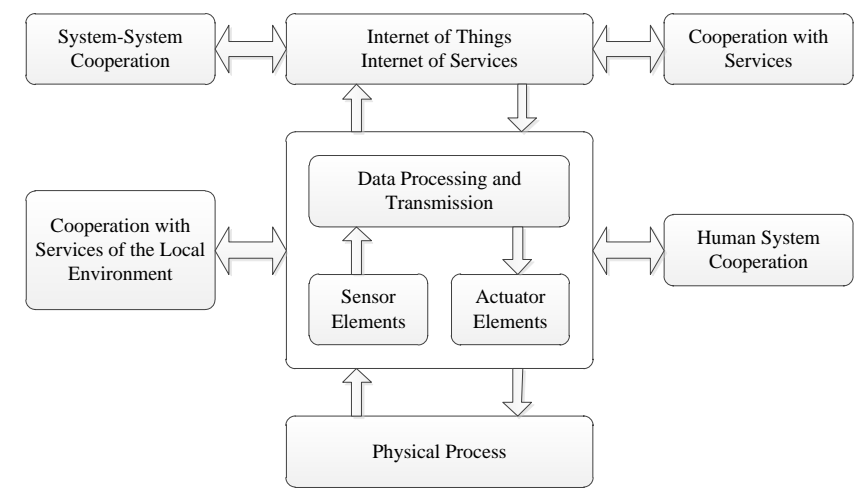

Fig. 1. Structure of cyber-physical systems [7]

\section{IIOT SUITE}

Nowadays IoT devices are ubiquitous as these devices can be found at smart factories, warehouses, offices etc. Intelligent and automated machines and equipment are examples of IoT devices which are used in manufacturing. The increased number and diversity of IoT devices, data exchange and communication as well as the need to manage different IoT devices present numerous challenges to the manufacturing industry. Therefore, we propose a conceptual design of IIoT Suite, as described in Fig. 2, so as to provide an information and operational infrastructure for industrialists to leverage emerging technologies such as IIoT so as to better manage, monitor and control existing and new IoT devices and to transform from traditional manufacturing, industrial and business processes to high-value-added production or services. The proposed IIoT Suite can also be adopted in existing smart factories to optimize management of smart equipment and IoT devices and to improve production efficiency so as to provide customers with innovative and high value-added production or services. 


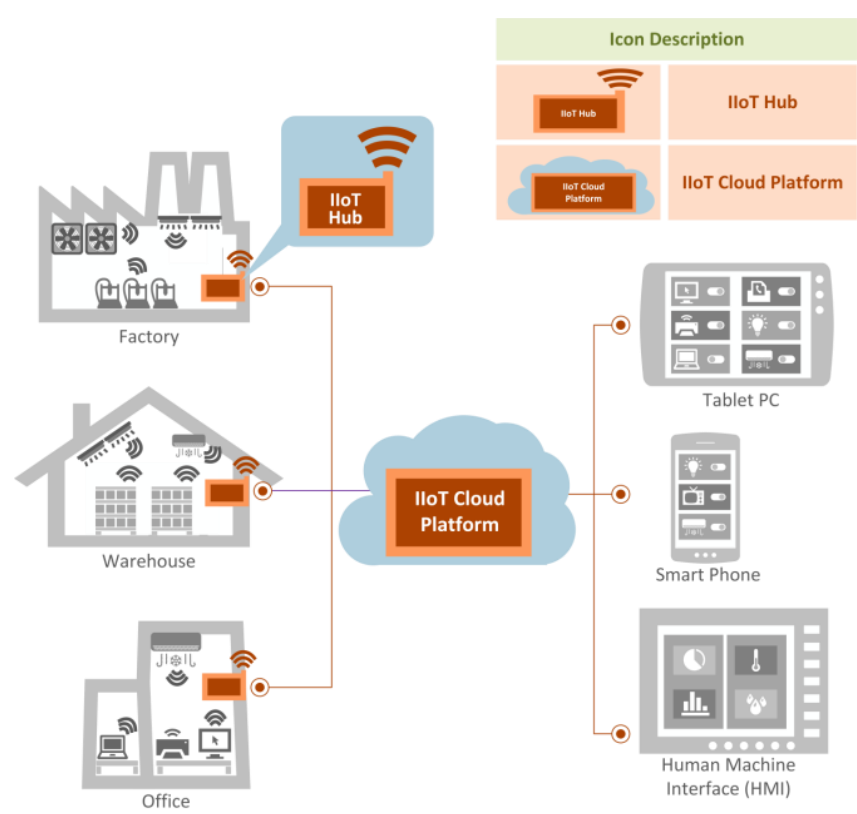

Fig. 2. Conceptual design of IIoT Suite

\section{A. IIoT System Architecture}

The proposed IIoT suite is constructed based on multi-tier system architecture as shown in Fig. 3. Different IoT devices such as intelligent and automated machines, equipment, etc. are connected with the IIoT-based Smart Hub through device APIs (Application Program Interfaces).

\begin{tabular}{|c|c|c|}
\hline $\begin{array}{c}\text { Manufacturing } \\
\text { Execution System } \\
\text { (MES) }\end{array}$ & $\begin{array}{c}\text { Automated Storage } \\
\text { and Retrieval System } \\
\text { (ASS/RS) }\end{array}$ & $\begin{array}{c}\text { Enterprise Asset } \\
\text { Management (EAM) } \\
\text { System }\end{array}$ \\
\hline \multicolumn{3}{|c|}{ XML/ ISON over MQ Messaging Service / RESTful Web Service } \\
\hline \multicolumn{2}{|c|}{$\begin{array}{l}\text { Microservices-based } \\
\text { IloT Cloud Platform }\end{array}$} & $\begin{array}{c}\text { NoSQL Database for } \\
\text { Big Data Analytics }\end{array}$ \\
\hline \multicolumn{3}{|c|}{ XML/ ISON over MQTT / COAP Lightweight Protocol } \\
\hline \multicolumn{3}{|c|}{$\begin{array}{l}\text { Industrial Internet of Things (IIoT)-based } \\
\text { Smart Hub }\end{array}$} \\
\hline \multicolumn{3}{|c|}{ XML/ ISON over MQTT / COAP Lightweight Protocol } \\
\hline loT Device ${ }_{1}$ & loT Device ${ }_{2}$ & loT Device ${ }_{n}$ \\
\hline Device API & Device API & Device API \\
\hline Equipment & Equipment & Equipment \\
\hline
\end{tabular}

Fig. 3. Schematic design of system architecture

The IIoT-based Smart Hub receives raw data from IoT devices; it then transfers the preprocessed data to the Microservices-based IIoT Cloud Platform through XML/JSON over the MQTT/CoAP Lightweight Protocol. The Micro-servicesbased IIoT Cloud Platform consists of a variety of small and independent services. Each micro-service has its own business logic and database and each service can operate on its own independently. Micro-service can allocate and utilize resources for different job tasks in a dynamic and flexible way. The platform can transfer useful statistical data or information to other Information Technology (IT)/Operation Technology (OT) systems such as Manufacturing Execution System (MES), Automated Storage and Retrieval System (AS/RS) and Enterprise Asset Management (EAM) System through XML/JSON over MQ Message Service and/or RESTful Web Service to support risk and process management.

\section{B. IIoT Smart Hub}

The IIoT-based Smart Hub is developed for managing IoT devices at distributed locations including smart factories, warehouses, and offices. In practical situations, wired communication is one of the reliable methods to transfer data to the backend platform. However, as different IoT devices may have their own communication methods such as $\mathrm{Wi}-\mathrm{Fi}$, Bluetooth/BLE, ZigBee and Z-Wave; it is inefficient to connect various IoT devices to the backend platform directly. The most adopted solution is to collect data from IoT devices by using their communication methods and then transfer data through wired communication.

To address the above mentioned problems, the IIoT-based Smart Hub is constructed, which can act as a gateway for existing IoT devices. The function of the IIoT-based Smart Hub is three-fold. First, it provides a simple and convenient method for data preprocessing, data exchange and communication between existing IoT devices and the backend platform, which means with the use of the IIoT-based Smart Hub, updates or enhancements of data exchange and communication channels can be realized. Second, it provides a sustainable solution to meet the challenges of future expansion of IoT devices. Third, the IIoT-based Smart Hub is designed to build a secure connection between the detected IoT devices and the Micro-services-based IIoT Cloud Platform.

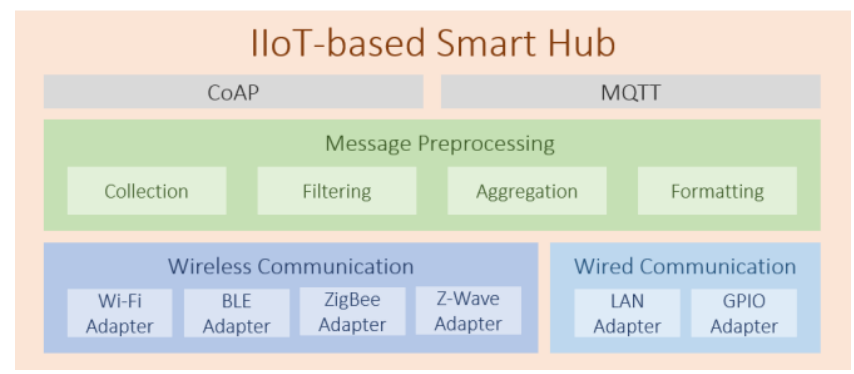

Fig. 4. Main components of IIoT-based Smart Hub

Fig. 4 shows the main components of IIoT-based Smart Hub. The IIoT-based Smart Hub is capable of communicating with IoT devices through wireless communications such as Wi$\mathrm{Fi}$, Bluetooth/BLE, ZigBee and Z-Wave or wired communications such as LAN and GPIO. Once the IIoT-based Smart Hub receives the data from IoT devices, it performs message preprocessing that includes data collection, filtering, aggregation and formatting. The IIoT-based Smart Hub transfers the preprocessed data to a Micro-services-based IIoT Cloud Platform through XML/JSON over the MQTT/CoAP Lightweight Protocol. Through establishing a secure connection between the IoT devices, the Micro-services-based IIoT Cloud Platform and IIoT-based Smart Hub, manufacturers 
and industrialists can simply select the relevant micro-services for the specific IoT devices on the platform.

\section{IIoT Cloud Platform}

The Micro-services-based IIoT Cloud Platform, acting as the brain for the IIoT Suite, is the most important output, which is designed based on small and independent services, named micro-services. Each micro-service has its own business logic and database, and it can operate individually. The microservices architecture allows users to control a single application rather than a whole platform. The micro-services architecture has many advantages over the traditional monolithic architecture. On the one hand, the use of various IoT devices is increasing exponentially; it becomes difficult to forecast the number and variety of IoT devices. When a traditional monolithic architecture platform is used, the whole platform has to be suspended for maintenance or upgrade. On the other hand, the micro-services architecture allows users to add, delete, update or enhance a particular service without affecting the operation performance of the whole platform which is beneficial for the maintenance processes. Moreover, it is also capable of optimizing platform usage and maintaining reliable platform performance. With the support from micro-services, the Micro-services-based IIoT Cloud Platform can offer a single platform with Graphical User Interfaces (GUI) for industrialists to communicate, manage, monitor and control IoT devices remotely in a simple, efficient and convenient way.

Fig. 5 shows the main components of a Micro-servicesbased IIoT Cloud Platform. Examples of the micro-services include Identity and Access Management (IAM), load balancing, devices/services discovery, devices configuration, routing/rule engine, monitoring and control. These services can communicate with each other through messaging channels and they perform small tasks independently. Due to various types and functions of the micro-services, each micro-service is allocated to an individual NoSQL (non-relational) database for data storage.

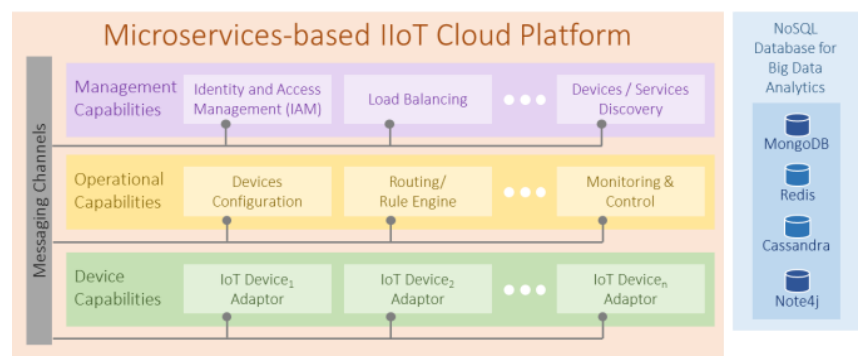

Fig. 5. Main components of IIoT Cloud Platform

The application scenarios of the Micro-services-based IIoT Cloud Platform can be described as follows. When a user would like to configure an IoT Device, the platform will check the user identify first by using the IAM micro-service. The requested micro-service can be performed only if the user has the appropriate permission. If a large number of users are requesting the same micro-service, not only the identities and permissions of these users are examined, the loading of the micro-service will also be checked. If the micro-service is overloaded, the micro-services architecture can allow the platform to replicate one or more instances of that micro- service instance to share the workload of the overloaded tasks. Once the loading of the micro-service is back to normal, the platform will kill excess micro-services. The micro-services architecture can allow the platform to optimize its usage according to the actual pattern of demand.

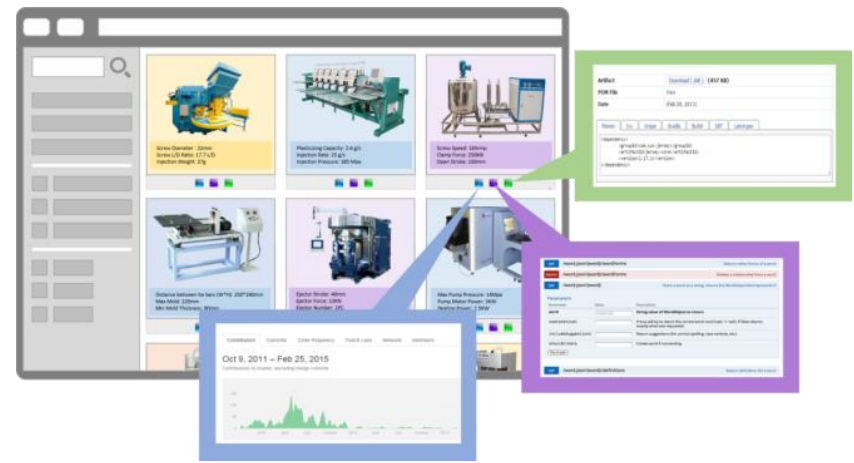

Fig. 6. Graphical user interface of IIoT Cloud Platform

The GUI between the Micro-services-based IIoT Cloud Platform and users is illustrated in Fig. 6. The platform provides a graphical presentation layout that can facilitate manufacturers and industrialists to manage, monitor and control the connected IoT devices located in different smart factories, warehouses and offices. Once a new IoT Device adaptor (e.g. IoT Device) is established on the Micro-servicesbased IIoT Cloud Platform and a new IoT device (e.g. machine) are connected through the IIoT-based Smart Hub, the platform is able to propose appropriate services for the identified IoT devices by using the devices/service discovery micro-service. The user or other third party applications can further execute or control the identified IoT device through the platform, the IIoT-based Smart Hub and appropriate IoT Device adapter (e.g. IoT Device).

\section{CONCLUSION}

To deal with rising labor wage rates and severe shortage of labor in China, an information and operational infrastructure, called IIoT Suite, is proposed, aiming to accomplish reindustrialization of Hong Kong and to upgrade or transform industrial operations so as to implement smart production and run high value-added manufacturing processes. In the IIoT Suite, the IIoT-based Smart Hub is developed as a gateway to simplify connection and communication with various IoT devices (e.g. intelligent and automated machines and equipment) so as to facilitate efficient data exchange and communication between IoT devices and the Micro-servicesbased IIoT Cloud Platform. The Micro-services-based IIoT Cloud Platform provides a reliable and convenient way for industrialists to communicate, manage, monitor and control IoT devices at distributed locations such as smart factories, warehouses and offices. The IIoT Suite presents a simple and convenient way to exchange data amongst existing or forthcoming IoT devices. It also provides a single platform for manufacturers and industrialists to manage, monitor and control of various connected IoT devices more effectively. Furthermore, the proposed IIoT Suite is capable of transferring useful statistics from the platform to other information and operation systems for further investigation and analysis. 


\section{ACKNOWLEDGMENT}

This work is supported by the Hong Kong Polytechnic University. Our gratitude is also extended to the research committee and the Department of Industrial and Systems Engineering of the Hong Kong Polytechnic University for support in this project (H-ZDAR) and (RTY0).

\section{REFERENCES}

[1] Office of the Chief Executive (2016), "Development of Industries" 2016 Policy Address, Retrieved on 19 January 2016 from http://www.policyaddress.gov.hk/2016/eng/p76.html

[2] The Government of the Hong Kong Special Administrative Region (2016), "Innovation and technology boosted in Policy Address", news.gov.hk, Retrieved on 5 February 2016 from http://www.ogcio.gov.hk/en/news and publications/legco briefs/201 5/12/doc/lb_20151214.pdf

[3] Innovation and Technology Bureau (2015), "Innovation and Technology Bureau established", Innovation and Technology Bureau, Retrieved on 5 February 2016 from http://www.itb.gov.hk/tc/speeches/2015/pr 20151120 3.htm
[4] Yau, E. (2015), "The manufacturers moving production from China back to Hong Kong", South China Morning Post, Retrieved on 19 January 2016 from http://www.scmp.com/lifestyle/article/1823459/manufacturersmoving-production-china-back-hong-kong

[5] H. Kagermann, J. Helbig, A. Helliger and W. wahlster, "Recommendations for Implementing the Strategic Initiative INDUSTRIE 4.0: Securing the Future of German Manufacturing Industry," Final Report of the Industrie 4.0 Working Group 2013, Forschungsunion

[6] J. Lee, B. Bagheri and H.A. Kao, "A Cyber-Physical Systems architecture for Industry 4.0-based manufacturing systems," Manufacturing Letters, 2015, vol. 3, pp. 18-23

[7] M. Mikusz, "Towards an Understanding of Cyber-physical Systems as Industrial Software-Product-Service Systems," Procedia CIRP, 2014, vol. 16, pp. 385-389.

[8] L. Monostori, "Cyber-physical Production Systems: Roots, Expectations and R\&D Challenges," Procedia CIRP, 2014, vol. 17, pp. 9-13 